\title{
Adaptation of Hybrid Human-Computer Interaction Systems using EEG Error-Related Potentials
}

\author{
Ricardo Chavarriaga, Andrea Biasiucci, Killian Förster, Daniel Roggen, \\ Gerhard Tröster and José del R. Millán
}

\begin{abstract}
Performance improvement in both humans and artificial systems strongly relies in the ability of recognizing erroneous behavior or decisions. This paper, that builds upon previous studies on EEG error-related signals, presents a hybrid approach for human computer interaction that uses human gestures to send commands to a computer and exploits uses brain activity to provide implicit feedback about the recognition of such commands. Using a simple computer game as a case study, we show that EEG activity evoked by erroneous gesture recognition can be classified in single trials above random levels. Automatic artifact rejection techniques are used, taking into account that subjects are allowed to move during the experiment. Moreover, we present a simple adaptation mechanism, that uses the EEG signal to label newly acquired samples that can be use to re-calibrate the gesture recognition system in a supervised manner. Offline analysis show that, although the achieved EEG decoding accuracy is far from being perfect, these signals convey sufficient information to significantly improve the overall system performance.
\end{abstract}

\section{INTRODUCTION}

Performance improvement in both humans and artificial systems strongly relies in the ability of recognizing erroneous behavior or decisions. A wealth of studies have been devoted to characterize neural activity correlated to erroneous actions or feedback [1], [2] and more recently, several works have focused on the use of the so-called error-related potentials in non-invasive brain-computer interfaces [3], [4]. Remarkably, besides showing the existence of these potentials during BCI operation it has also been shown that their detection can be used to correct erroneous BCI decisions [5], or to guide learning of adapting artificial systems [6].

These studies have been typically performed during control of simulated devices where the subject is asked to limit its movements in order to avoid artifact contamination of the EEG signals. It is therefore, not yet clear whether this type of signals can be detected or exploited in less restrictive conditions. One of the goals of this work is to address precisely this issue. Moreover, the idea of hybrid systems i.e. based on different communication channels- has been put forward recently as a way to improve the performance and usability of BCI systems and neuroprostheses [7]. We argue that the hybrid approach can exploit the brain activity to convey information about the subject cognitive and perceptual state, while control commands can be delivered using faster,

R. Chavarriaga, A. Biasiucci and J. del R. Millán are with EPFL, Chair on Non-Invasive Brain-Computer Interface (CNBI), CH-1015 Lausanne, Switzerland. \{name.surname\} depfl.ch

K. Förster, D. Roggen and G. Tröster are with ETH Zurich, IFE, Wearable Computing Lab, CH-8092 Zurich, Switzerland. ffoerster, roggen, troester\}eife.ee.ethz.ch more efficient channels (e.g. residual muscular activity). Along these lines, in this work we study the possibility of decoding EEG error-related signals during human computer interaction, and the use of such signals to improve the performance of the artificial interactive system. As a case study we use a gesture-based interaction system where hand gestures are used to deliver commands to a computer and brain signals are used to implicitly obtain information about the perceived performance of the interactive system. This information can then be used to improve the performance of the gesture recognition system.

\section{METHODS}

\section{A. Experimental setup}

We study error-related EEG potentials elicited during gesture-based human-computer interaction (HCI). Specifically, this experiment allows to evaluate brain signals elicited by wrong recognition of gestures, and assess whether these signals can be used to assess the performance of the HCI system and adapt its behavior. During the experiment subjects played a computerized version of a "memory game" consisting of 8 image pairs (Figure 1). Images are randomly distributed in a four by four matrix and hidden behind question marks. The subjects have to find identical pairs of images, which are then removed from the screen. The game is finished when all image pairs were correctly found. Subjects control a cursor to select and flip the images, using five hand gestures (left, right, up and down hand movements shift the image selection cursor; while closing the hand flips the image). Each directional gesture starts and ends at a central home position. $500 \mathrm{~ms}$ after the gesture finishes the game action is executed providing feedback about whether the command was correctly recognized by the $\mathrm{HCI}$ interface.

During recordings hand gestures were decoded using a light barrier frame and a reed switch in the hand for the closing gesture, ensuring accurate gesture recognition. Additionally, hand acceleration was also recorded using a triaxial acceleration sensor at the subjects fingertips sampled at $64 \mathrm{~Hz}$. This data was used offline to assess EEG-based adaptation of gesture recognition systems. More details about the experimental setup can be found in [8]

\section{B. Experimental protocol}

Seven healthy male subjects aged 25 to 47 took part in the the experiment. It is composed of 7 sessions, each corresponding to two full memory games. The duration of each game ranges from three to five minutes, and a 


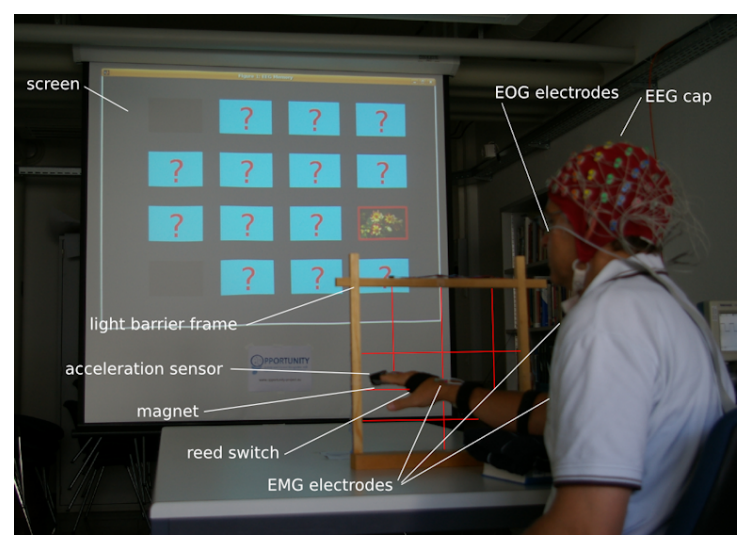

Fig. 1. Experimental setup showing the computer game presented on the screen; the light-barrier frame, magnet and reed switches capture game control gestures. Simultaneously acceleration, EMG (right wrist, biceps and shoulder), EOG, and EEG are recorded for offline analysis.

short pause is done between two consecutive games. During each game, accurate gesture recognition is provided by the light-barrier frame and errors are artificially induced in the feedback presented to the subject. Error rates goes from 5\% to $33 \%$. In the case of added errors, the user command is replaced by another command selected at random. The overall recording for a subject contains around 2700 gestures, and its total duration was about 2 hours.

During the whole experiment we recorded 64 channel EEG activity using a Biosemi ActiveTwo system with a sampling rate of $2048 \mathrm{~Hz}$, then downsampled to $512 \mathrm{~Hz}$. Signal was spatially filtered using common average reference and bandpass filtered in the $1-10-\mathrm{Hz}$ range. Muscular activity in the wrist, biceps and deltoid, as well as horizontal EOG (outer canthi) was also recorded using the same system.

Since subjects have to move during the experiment and the visual cursor feedback may trigger eye movements, we apply an automated EOG artifact correction method based on regression analysis [9]. To this end, a short calibration session (approximately 90 seconds) is recorded previous to the experiment where the subject is asked to perform circular eye movements and eye blinks. Data from this session is used to estimate correction function based on the measured EOG and EEG signals. Due to technical problems in the recording of the calibration data of subject 2 , this subject was not taken into account in the remaining study.

\section{Classification using Bayesian filtering}

We classify EEG activity after the feedback as corresponding to an error or correct recognition of the gesture. We rely on a Bayesian filtering technique so as to take into account the time course of the evoked signal, while providing a probabilistic output that can be used as a measure of the reliability of the estimated state [10]. This technique is based on recursive Bayesian estimations, where at each time step the state estimation (i.e. output class) is updated based on the observations and the previous state estimation. The observations correspond to the EEG activity at the current time sample and the states correspond to the output class, i.e. an erroneous or correct recognition of the gesture. Based on previous studies EEG classification is based on the activity of the $\mathrm{FCz}$ and $\mathrm{Cz}$ electrodes in the window [200, 400] ms.

We defined two possible states $S_{t} \in[0,1]$ for correct and error classes, respectively; and the observation vector $O_{t}=\left[F C z_{t}, C z_{t}\right]$, is composed of the activity of the corresponding electrodes at time t. States and observations for a given trial are respectively denoted $S_{0: T}$ and $O_{0: T}$, where $t=0$ corresponds to the feedback onset and $\mathrm{T}$ the final time sample. We defined a transition model as a first order Markov model over time,

$$
P\left(S_{t} \mid S_{0: t-1}\right)=P\left(S_{t} \mid S_{t-1}\right)=\mathcal{I}
$$

for $t=1 \ldots T$. This transition model is equal to the identity since the state does not change within a trial. In addition we define a sensor model, $P\left(O_{t} \mid S_{t}\right)$, predicting the observations given the state. This two expressions yield the joint probability,

$P\left(S_{0: T} O_{0: T}\right)=P\left(S_{0}\right) P\left(O_{0} \mid S_{0}\right) \prod_{t=1}^{T}\left(P\left(S_{t} \mid S_{t-1}\right) P\left(O_{t} \mid S_{t}\right)\right)$

Then for a sample $t$ in a trial we can estimate its state by recurrently predicting the state using the transition model (3), and then estimate the state based in the sensor model (4) as follows,

$$
\begin{gathered}
P\left(S_{t} \mid O_{0: t-1}\right)=\sum_{S_{t-1}}\left(P\left(S_{t} \mid S_{t-1}\right) P\left(S_{t-1} \mid O_{0: t-1}\right)\right) \\
P\left(S_{t} \mid O_{0: t}\right) \propto P\left(O_{t} \mid S_{t}\right) P\left(S_{t} \mid O_{0: t-1}\right)
\end{gathered}
$$

Given the identity transition matrix, the predictionestimation recurrent calculus is simplified,

$$
P\left(S_{t}=1 \mid O_{1: t}\right) \propto P\left(O_{t} \mid S_{t}\right) P\left(S_{t-1}=1 \mid O_{1: t-1}\right)
$$

and correspondingly for $P\left(S_{t}=0 \mid O_{1: t}\right)$. A trial is then assigned to the class with the highest probability.

Estimations from both channels are combined using a naive fusion, $P\left(O_{t} \mid S_{t}\right)=P\left(F C z_{t} \mid S_{t}\right) P\left(C z_{t} \mid S_{t}\right)$. The sensor model $P\left(O_{t} \mid S_{t}\right)$ is defined by a Gaussian distribution with a mean $\mu_{t}$ and a variance $\sigma_{t}^{2}$, estimated using the training dataset. The recurrent nature of this technique allows the updating of the estimated state probability as new samples are available, making it suitable for real-time applications.

\section{EEG based adaptation of activity recognition}

We assess theoretically the feasibility of using the errorrelated potentials to improve the performance of the gesture recognition system. In particular, we assume that a subject independent gesture classifier has been previously obtained and the EEG decoding signals will be used to adapt this classifier to a specific new user. Accordingly, trials that not classified as errors would signal that the last gesture was 
correctly recognized, it can thus be used as an example to further train the current classifier in a supervised manner [8].

Using the recorded data, we build offline a kNN gesture classifier $(\mathrm{k}=13)$ using hand acceleration as input and information from the light-barrier frame as ground truth. An initial user-independent gesture classifier is trained using data from 6 of the subjects (480 randomly selected training samples equally distributed across classes). Adaptation is then tested in the remaining subject using incremental online learning [11]. Namely, each trial not eliciting ErrPs is assumed correct and included in a new training set for recalibration of the $\mathrm{kNN}$ classifier. Data from the remaining subject is divided into an adaptation and validation set (approx 2000 and 500 samples, respectively) and different levels of EEG accuracy were simulated. This simulation gives us an estimation of the potential user adaptation of a general recognition system, and can be used to estimate the possible improvement given the EEG classification accuracy of the Bayesian filter classifier described above. Reported results correspond to the average over 20 repetitions across all subjects.

\section{RESULTS}

\section{A. Event-related potentials}

Figure 2 shows correction coefficients obtained using the regression technique. This correction is applied to the EEG activity recorded while playing the game before computing the ERPs and testing single trial classification. Topographical representation of the corrected scalp activity after the feedback is presented in the same figure (Grand average for the error minus correct condition). It shows negative activity at fronto-central medial sites around $200 \mathrm{~ms}$, followed by a positive activation in that region around $350 \mathrm{~ms}$. Accordingly, grand average ERP on the $\mathrm{FCz}$ electrode over all subjects shows a positive deflection peaking about 350 ms. In most subjects the ERP shows a preceding, smaller negative deflection between 200 and $300 \mathrm{~ms}$. although this component is not well synchronized across subject. This activity profile is consistent with previous experiments on error-related potentials in brain computer interfaces that have also reported ERPs composed by a negative deflection after $200 \mathrm{~ms}$ followed by a larger positivity after $300 \mathrm{~ms}$.

\section{B. Classification}

Single trial classification performance was assessed using three recording sessions as testing set. The average size of the testing set was 939 trials, and the average rate of error trials was $27.1 \%$. The classification performance using the Bayesian filter described in section II-C is shown in Figure 3(a). In this figure, sensitivity corresponds to the rate of true positives (i.e. error trials correctly classified), while the specificity corresponds to the rate of true negatives (i.e. properly classified correct trials). It can be seen that the classifier performance is above random level for all subjects with the exception of subject 7. Figure 3(b) shows the average estimated state $P\left(S_{t}\right)$ for error and correct trials (red and green traces, respectively). Consistently with the classification results, on average the estimated state of
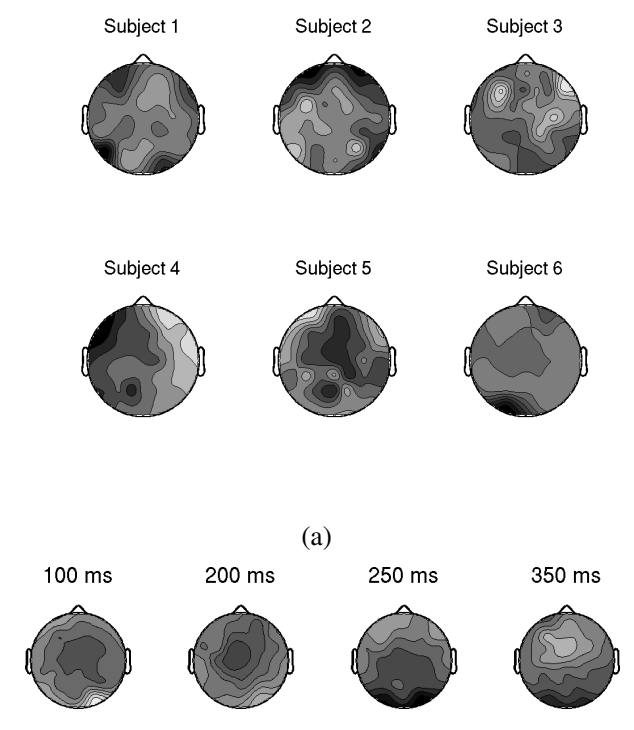

(b)

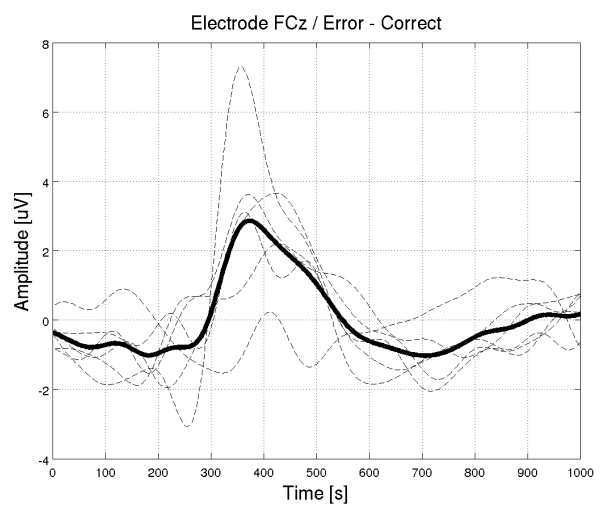

(c)

Fig. 2. (a) EOG correction coefficients obtained using the regression technique. (b) Topographical representation of scalp activity at different time points after the feedback. (c) Event-related potentials at $\mathrm{FCz}$ electrode (error minus correct). Single subject ERP is shown in thin lines; Solid line, average of all subjects. Topographical maps are color coded from black to white ranging from negative to positive values, respectively.

error trials is greater than that of correct ones (notice that $P\left(S_{t}\right)=1$ corresponds to the error class).

\section{Adaptation}

Figure 3(c) shows the theoretical assessment subjectdependent adaptation for different performance levels of the EEG classification. Gray level maps show the relative improvement in accuracy for the different regions of the ROC space, as well as the number of subjects for which this improvement is statistically significant when compared to the subject-independent gesture recognition system $(p<0.05)$. EEG classification accuracy for all subjects is superimposed showing that although the performance is not very high, EEG signals convey enough information to significantly increase the performance of the gesture recognition system for most subjects. In the present case the maximal recognition improvement is around $6.4 \%$, while the maximal possible improvement (i.e. with perfect EEG recognition) is of $16.8 \%$. 


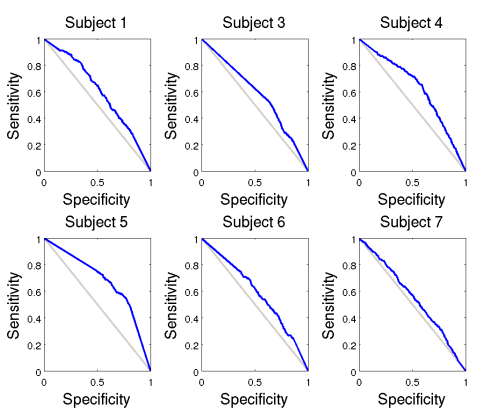

(a)

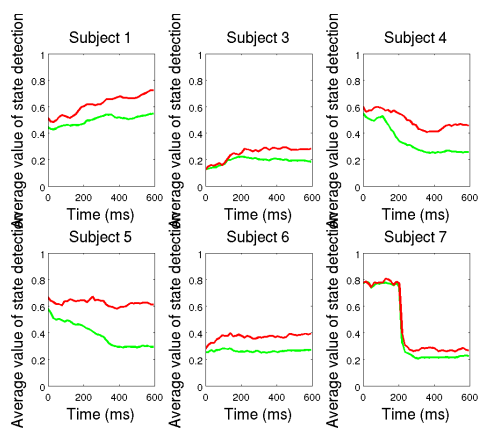

(b)

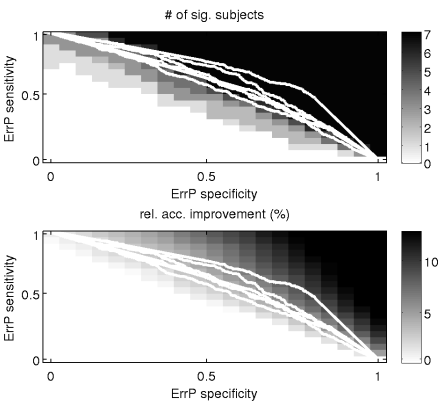

(c)

Fig. 3. Classification of error-related potentials. (a) Classification performance in the ROC space. Dashed and solid lines show the classifier accuracy with and without rejection. (b) Average estimated state for error and correct trials (red and green respectively). (c) EEG based adaptation of gesture recognition system. Each plot shows the potential improvement in gesture recognition for different EEG classification performance in the ROC space.

\section{CONCLUSIONS AND FUTURE WORKS}

In this work we have focus on the detection of brain activity elicited by errors in human computer interaction. Following a hybrid approach where hand-gestures are used to send commands to a computer game, the EEG activity is decoded to recognize perceived errors in the command interpretation. Single trial classification using Bayesian filtering performs above random levels for most of the tested subjects, using activity of fronto-central electrodes traditionally used for recognition of error-related potentials. Moreover, using the decoded EEG signal to label new samples, it is possible to re-train the gesture recognition system so as to adapt to new users. Off-line analysis of this adaptation process shows that significant accuracy improvement can be achieved with the EEG classification performance reported here.

The present study constitute an example of a hybrid system where user movements are combined with cognition-related feedback decoded from EEG. Contrasting with previous studies where subjects where explicitly asked to limit their movements, such restriction does not exist in this experiment. In the present study an automatic artifact removal technique is used to reduce the signal contamination. Future work will be devoted to further explore this and other denoising techniques (e.g. ICA) that may allow the analysis of EEG signals in less constrained scenarios, thus enabling characterization of neural responses during natural interaction [12], and the deployment of hybrid BCI systems outside research laboratory conditions. In addition, further studies will also performed to assess the influence of error rate in the evoked EEG activity and its classification, as well as the online application of the presented framework.

\section{ACKNOWLEDGMENTS}

This work has been supported by the EU-FET project ICT-225938 Opportunity: Activity and Context Recognition with Opportunistic Sensor Configuration and by the EUICT project FP7-224631 TOBI: Tools for Brain-Computer Interaction. This paper only reflects the authors' views and funding agencies are not liable for any use that may be made of the information contained herein.

\section{REFERENCES}

[1] M. Falkenstein, J. Hoormann, S. Christ, and J. Hohnsbein, "ERP components on reaction errors and their functional significance: A tutorial." Biol Psychol, vol. 51, no. 2-3, pp. 87-107, Jan 2000.

[2] S. F. Taylor, E. R. Stern, and W. J. Gehring, "Neural systems for error monitoring: Recent findings and theoretical perspectives." Neuroscientist, vol. 13, no. 2, pp. 160-172, Apr 2007. [Online]. Available: http://dx.doi.org/10.1177/1073858406298184

[3] L. C. Parra, C. D. Spence, A. D. Gerson, and P. Sajda, "Response error correction-A demonstration of improved human-machine performance using real-time EEG monitoring." IEEE Trans Neural Syst Rehabil Eng, vol. 11, no. 2, pp. 173-177, June 2003.

[4] G. Schalk, J. R. Wolpaw, D. J. McFarland, and G. Pfurtscheller, "EEG-based communication: Presence of an error potential." Clin Neurophysiol, vol. 111, no. 12, pp. 2138-2144, Dec 2000.

[5] P. W. Ferrez and J. Millán, "Simultaneous real-time detection of motor imagery and error-related potentials for improved BCI accuracy," in Proc 4th Intl. Brain-Computer Interface Workshop and Training Course, Graz, Austria, September 2008.

[6] R. Chavarriaga, P. W. Ferrez, and J. Millán, "To err is human: Learning from error potentials in brain-computer interfaces," in Int Conf Cognitive Neurodynamics, R.Wang, F. Gu, and E. Shen, Eds., Shanghai, China, 2007, pp. 777-782. [Online]. Available: http://www.iccn2007.org/

[7] B. Z. Allison, E. W. Wolpaw, and J. R. Wolpaw, "Brain-computer interface systems: Progress and prospects." Expert Rev Med Devices, vol. 4, no. 4, pp. 463-474, Jul 2007. [Online]. Available: http://dx.doi.org/10.1586/17434440.4.4.463

[8] K. Förster, A. Biasiucci, R. Chavarriaga, J. d. R. Millán, D. Roggen, and G. Tröster, "On the use of brain decoded signals for online user adaptive gesture recognition systems," in Pervasive 2010 The Eighth International Conference on Pervasive Computing, 2010. [Online] Available: http://www.pervasive2010.org/

[9] A. Schlögl, C. Keinrath, D. Zimmermann, R. Scherer, R. Leeb, and G. Pfurtscheller, "A fully automated correction method of EOG artifacts in EEG recordings." Clin Neurophysiol, vol. 118, no. 1, pp. 98-104, Jan 2007. [Online]. Available: http://dx.doi.org/10.1016/j.clinph.2006.09.003

[10] J.-M. Bollon, R. Chavarriaga, J. d. R. Millán, and P. Bessière, "EEG error-related potentials detection with a Bayesian filter," in Proc. 4th International IEEE/EMBS Conference on Neural Engineering NER '09, 2009, pp. 702-705.

[11] D. W. Aha and D. Kibler, "Instance-based learning algorithms," in Machine Learning, 1991, pp. 37-66.

[12] S. Makeig, K. Gramann, T.-P. Jung, T. J. Sejnowski, and H. Poizner, "Linking brain, mind and behavior." Int J Psychophysiol, vol. 73, no. 2, pp. 95-100, Aug 2009. [Online]. Available: http://dx.doi.org/10.1016/j.ijpsycho.2008.11.008 